\title{
A NOTE ON THE DIFFERENTIAL FORMS ON EVERYWHERE NORMAL VARIETIES
}

\author{
YÛSAKU KAWAHARA
}

A. Weil proposed in his book "Foundations of algebraic geometry" several problems concerning differential forms on algebraic varieties. S. Koizumi" has proved that if $\omega$ is a differential form on a complete variety $\mathbf{U}$ without multiple point, which is finite at every point of $U$, then $\omega$ is the differential form of the first kind. The following example shows that on everywhere normal varieties with multiple points this statement holds no more; that is: $A$ differential form on a everywhere normal variety which is finite on eyery simple point of its variety is not always the differential form of the first kind.

In the projective space of dimension 3 with the field of characteristic 0 as universal domain, we consider the variety $V^{2}$ with homogeneous equation $X_{3}^{4}$ $=X_{1}^{4}+X_{2}^{4}$. Let $k$ be a defining field of $\mathrm{V}$ and $\left(x_{0}, x_{1}, x_{2}, x_{3}\right)$ a set of homogeneous coordinates of a generic point $\mathrm{P}$ of $\mathrm{V}$ over $k$.

1) Put $\frac{x_{1}}{x_{0}}=x, \frac{x_{2}}{x_{0}}=y, \frac{x_{3}}{x_{0}}=z ; \frac{x_{0}}{x_{1}}=u, \frac{x_{3}}{x_{1}}=v, \frac{x_{3}}{x_{1}}=w$.

Since $k[1, x, y, z], k[u, 1, v, w], k\left[x_{0} / x_{2}, x_{3} / x_{3}, 1, x_{3} / x_{2}\right], k\left[x_{0} / x_{3}, x_{1} / x_{3}, x_{2} / x_{3}, 1\right]$ are integrally closed, $\mathbb{V}$ is everywhere normal. And it is easily seen that $(1,0,0,0)$ is the only singular point of $\mathrm{V}$.

2) Consider the differential form $\omega=1 / z^{3} d x d y$ on $V$ defined over $k$; $\omega$ is finite on every point of $\mathrm{V}$ except $(1,0,0,0)$.

$$
\begin{gathered}
z^{3} d z=x^{3} d x+y^{3} d y \\
\frac{1}{z^{3}} d x d y=+\frac{1}{y^{3}} d z d x=\frac{1}{x^{3}} d x d z=-\frac{1}{2 v^{3}} d u d v=\frac{1}{2 v^{3}} d u d w \text { etc. } \\
w^{4}=1+v^{4} .
\end{gathered}
$$

This shows the assertion.

3) $\omega$ is not the differential form of the first kind.

Put $x=r, y / x=s, z / x=t$.

$$
k(\mathbf{P})=k(r, s, t) .
$$

Received Oct. 15, 1950.

1) On the differential forms of the first kind on algebraic varieties. Journal of the Mathematical Society of Japan. Vol. 1 (1949). 
On the locus $U$ of $(r, s, t)$ over $k$ the point $(0,0,1)$ is the simple point of $U$ with uniformizing parameters $r, s$.

$$
\frac{1}{z^{3}} d x d y=\frac{1}{r^{2} t^{4}} d r d s .
$$

$\frac{1}{r^{2} t^{3}}$ is not in the specialization ring of $(0,0,1)$ in $k(r, s, t)$.

Mathematical Institute,

Nagoya University 